\title{
Class Code
}

National Cancer Institute

\section{Source}

National Cancer Institute. Class Code. NCI Thesaurus. Code C82501.

A sequence of characters that represents a particular category of items. 